\title{
SIMULATION IMPROVES EFFICIENCY AND QUALITY IN SHOE MANUFACTURING
}

\author{
Mohan Mano Hassan $^{(a)}$, Sai Priyanka Kalamraju ${ }^{(a)}$, Sandeep Dangeti ${ }^{(a)}$, Sirisha Pudipeddi $^{(a)}$, Edward J Williams $^{(\text {a) }}$

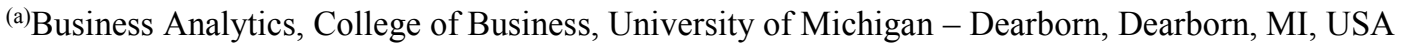 \\ ${ }^{(a)} \underline{\text { mohmano@umich.edu, }},{ }^{(a)}$ kalamraj@umich.edu, ${ }^{\left({ }^{a}\right)}$ dvbittu@umich.edu, ${ }^{(a)}$ sirishap@umich.edu, ${ }^{(a)}$ williame@umich.edu
}

\begin{abstract}
Discrete-event process simulation now has a long and distinguished history of supporting the improvement of manufacturing processes. From those origins, it has expanded its applicability to supply chains, service industries, health care, and public transport. In manufacturing contexts, simulation modeling and analysis regularly helps fine-tune the trade-off between high inventory versus danger of stockout, improve and balance machine utilization, schedule workers more effectively, and improve performance metrics such as average and maximum times in queue and average and maximum length of queues. In the present work, the authors describe a successful application of simulation to the manufacture of footwear. The original manufacturing process was beset by problems including low throughput, high headcount, overly high or low machine utilization, unduly large rejection rates, and ergonomic concerns.

The simulation and analysis project described in this paper guided significant improvements, including doubling the output while reducing worker headcount to two-thirds of its initial value.
\end{abstract}

Keywords: discrete-event process simulation, footwear manufacture, resource utilization, queueing system performance metrics

\section{INTRODUCTION}

Historically, discrete-event process simulation was first and very extensively used to improve manufacturing operations; indeed, examples in the literature are numerous. A comprehensive review of progress and challenges in manufacturing simulation appears in (Mourtzis, Doukas, and Bernidaki 2014). There are many potential ways to improve a generic manufacturing operation; e.g., lower inventory while keeping stockouts very rare, improve queue performance metrics (especially average and maximum time in queues, and average and maximum length of queues), keep utilization of expensive equipment high while improving those queue performance metrics, use headcount more efficiently and less extravagantly, and increase throughput. Examples of such simulations abound; for example, (Mirzapourrezaei et al. 2011) applied simulation to improve an assembly process for manufacture of starters.
In the present work, simulation analysis was applied to a manufacturing plant in India which makes shoes. The global footwear market is, according to Allied Market Research, expected to garner $\$ 371.8 \mathrm{~B}$ by 2020 . This market is lively, expanding, and volatile, and includes both primary and secondary marketplaces (Weinswig 2016). Footwear manufacture has expanded globally; already prominent in the United States and China, it is rapidly entering India. In this paper, we present a successful simulation study of a shoe manufacturing plant in India. The factory management, already confronting the need to significantly increase production, and having accepted the necessity of payroll and equipment investments to do so, wanted guidance on the best way to proceed in this endeavor. This study has precedents: (Solomon, Jilcha, and Berhan 2014) used simulation to improve lead time prediction at a shoe manufacturer in Ethiopia; (Eryilmaz et al. 2012) analyzed production processes in a shoe manufacturing factory using simulation. The management of this plant presented significant concerns including relatively low throughput relative to high labor headcount, unbalanced utilization of equipment, long queues and waiting times therein, ergonomic concerns, and low throughput.

In the following sections, we (1) present an overview of the shoe-manufacturing operations, (2) describe the collection and analysis of input data, (3) discuss the building, verification, and validation of the simulation model, (4) highlight key results from this model obtained by output analysis, and (5) present conclusions, recommendations to the manufacturing plant's management, and the directions in which likely future work may proceed.

\section{OVERVIEW OF SHOE MANUFACTURING OPERATIONS}

The overall process of manufacturing shoes comprises the following high-level steps:

1. Procurement of raw materials

2. Cutting materials into shapes specified by design

3. Sewing

4. Assembly of parts

5. Finishing

6. Quality verification

7. Repair/rework or scrap as necessary

8. Packaging and shipping 
Repair work may result in scrapping the shoe or selling it at a loss ("seconds"). In this model, manufacture of shoes in the four most common sizes (seven, eight, nine, and ten inches) is considered. Fundamentally, a shoe has four basic components, as shown in Figure 1 below:

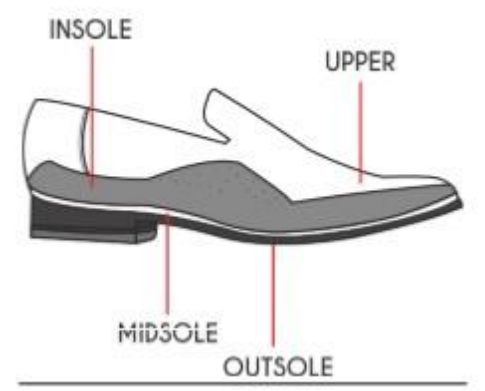

Figure 1: Basic Components of a Shoe

As configured at the beginning of this simulation study, the manufacturing facility comprises 93 workers and 88 worker-run (e.g., manual or semi-automatic, not automatic) machines. Open nine hours per day (9:00am to $6: 00 \mathrm{pm}$ ), the plant produces approximately 225 pairs of shoes daily. Informal industry norms indicate a plant of this size and capitalization investment "ought" to produce approximately 350 pairs of shoes per day. Additionally, manual transfer of shoes from one task to the next is currently giving rise to ergonomic concerns in particular, cervicobrachial (relating to the neck and arm) disorders, especially on the left side of the body; the importance and etiology of such disorders is documented in (Sällström and Schmidt 1984). Accordingly, the plant management wished to evaluate the feasibility of conveyors to move the shoes.

\section{DATA COLLECTION AND ANALYSIS}

Various essential input data was willingly and promptly provided to the analysts by plant supervisors and managers in Vellore, India. Arrival rates were 200 per hour during all but the fourth, eighth, and ninth hours of a standard nine-hour shift, with scheduled working time (i.e., less breaks and lunch) of eight hours. Average machine cycle times were provided as shown in Table 1 immediately below, all gathered from raw data.

Table 1: Mean Cycle Time of Operations

\begin{tabular}{|l|c|c|}
\hline $\begin{array}{l}\text { Process } \\
\text { (Machine) }\end{array}$ & $\begin{array}{l}\text { Avg (min) } \\
\text { Time/Piece }\end{array}$ & Capacity \\
\hline Cutting & 1.26 & Variable \\
\hline Sewing & 10.04 & Variable \\
\hline Assembling & 10.01 & Variable \\
\hline Inspection & 1.50 & 3 \\
\hline Repairing & 116.7 & 1 \\
\hline
\end{tabular}

After consultation with plant managers, the following assumptions were agreed to:

1. No maintenance is performed during the production period (it is done as necessary offshift, e.g., at night)
2. At the start of a day's production, sufficient raw material for the day is always available

3. No power interruptions occur

4. Downtimes at the cutting machines occur approximately monthly and take several hours to repair

5. Transportation time is not included in machine cycle time, and is small relative thereto (the longest transport distance in the plant being only ten meters)

6. All workers are assumed equally skilled, and hence produce the same quantity and quality in equal time

7. $2 \%$ of production is unsatisfactory and hence sent to repair; of the production sent there, $90 \%$ can be repaired and sold as "seconds;" the remaining $10 \%$ must be scrapped

Raw data was examined with the Stat::Fit ${ }^{\circledR}$ software (Benneyan 1998). After doing so, and with the precaution of showing plant managers and supervisors the characteristic histogram of distributions, arrivals were modeled as exponential, whereas machine cycle times were modeled as uniform or triangular distributions. As often occurs in practical simulation work, the value added by using distribution-fitting software was not so much advice on the best distribution to use (if indeed there existed a single "best" distribution), but rather cautionary advice on conspicuously inappropriate distributions to avoid (in these cases, the normal, gamma, and lognormal distributions).

\section{MODEL DEVELOPMENT, VERIFICATION,} AND VALIDATION

The very first model built was entirely conceptual - a flow chart of all paths taken from raw material to completed product: "Now, let put this shoe on your foot, and we'll see if it fits." Drawing and revising this model ensured that the analysts' understanding of the process flows exactly matched the expert knowledge of the plant managers and supervisors. Members of the project team then concurred in the choice of the Simio ${ }^{\circledR}$ software [SIMulation with Intelligent Objects] (Prochaska and Thiesing 2017), (Smith, Sturrock, and Kelton 2018) to construct a model of the salon's operations. Simio ${ }^{\circledR}$ provides constructs such as the Server (to model, for example, the cutting machines for the uppers, middle soles, and "outsoles"), the Worker (design checkers and quality checkers), a Combiner (e.g., gathering and sewing matched components together, and Conveyors (all but one proposed conveyor is to be nonaccumulating). These Simio $\AA$ constructs were used to model the key steps outlined in Table 1:

1. Cutting: the raw materials are cut into both left and right shoes in various sizes. Since the different parts of a shoe are made of different materials, the model considers three different procurement centers for cutting each shoe component. 
2. Sewing: The cut components are sent to the assembly section of the manufacturing unit for further processing - all sewing is performed in this phase for all shoe sizes, and for both left and right shoes.

3. Assembly: Unites all parts of the shoe.

4. Inspection: A quality-checking worker examines each shoe for defects.

5. Repair: Low-quality shoes may be sent to rework, if repairable, will be sent to rework. There, they will be repaired for sale at a discounted price. Shoes which cannot feasibly be repaired are scrapped (c.f. assumption 7 above).

The first iteration of the Simio ${ }^{\circledR}$ model deliberately contained only the Simio ${ }^{\circ}$ default values for parameters such as interarrival times and cycle times. Only after verification of correct entity flow in the animation were the values obtained from data collection and analysis (previous section) inserted. A partial two-dimensional screen shot of this model, as completed, is shown in Figure 2, Appendix. From this screen, a threedimensional animation is only two clicks away.

Verification and validation of the model used the following traditional and time-tested techniques (Sturrock 2018):

- Structured walkthroughs among the analyst team

- Sending one entity (arriving raw material) through the model and tracking, on a time line, every step taken by that entity

- Temporary removal of all stochastic variation of the model, followed by arithmetic checks using Microsoft Excel $\AA$

- Ensuring that every routing path placed in the model has non-zero traffic

- Directional variation (e.g., do queue lengths and waiting times increase when the cycle time of a machine is increased -- do they decrease when the capacity of a machine is increased?)

The usual errors were detected and corrected. Indeed, one error resulted in "pairs" of shoes comprising two left shoes or two right shoes! Another error, a routing logic error, was exposed by noticing that one of the routes in the model was never used. After correcting these errors was completed, comparison of model results, pertaining to the current system, with data actually observed during production yielded agreement of performance metrics within $4 \%$, helping the model achieve credibility with the plant management.

\section{EXPERIMENTATION AND RESULTS}

In keeping with actual policy at the manufacturing plant (production started anew daily), the model was run as a terminating system, hence zero warm-up time. Each replication represented one day's production. Since the facility typically works 25 days per calendar month, and after ensuring that 25 replications yielded sufficiently narrow confidence intervals for performance metrics, the three experimental scenarios were each run for 25 replications. The variance reduction technique (VRT) of common random numbers (CRNs) was used (Nakayama 2003). Simio ${ }^{\circledR}$ conveniently accommodates this technique by allowing the specification of different random number streams for interarrival times, cycle times, times to next failure, and repair times, as was done in this model. Confidence levels for estimation of performance metrics were taken at $95 \%$, and similarly for the confidence level of testing the null hypothesis of equal performance of any two scenarios. Each of these scenarios substituted short conveyors for currently manual transfers. Since management was proposing adding machines as well as workers, the key scenarios addressing one of the major investment decisions to be taken were listed as follows in Table 2:

Table 2. Investment Scenarios Considered

\begin{tabular}{|l|c|c|}
\hline Proposal & $\begin{array}{c}\text { \#sewing } \\
\text { employees }\end{array}$ & $\begin{array}{c}\text { \#assembly } \\
\text { employees }\end{array}$ \\
\hline Smaller invest & 27 & 20 \\
\hline Moderate invest & 30 & 25 \\
\hline Larger invest & 33 & 30 \\
\hline
\end{tabular}

The "moderate" scenario proved the best in this simulation experiment, relative to the performance metrics of machine utilizations, average time in system of a shoe, and most importantly, shoes output per day. The parameters and performance of the current system versus the scenario of moderate investment showed, as summarized in Table 3 immediately below:

Table 3. Proposed System Versus Current System

\begin{tabular}{|l|l|}
\hline \multicolumn{1}{|c|}{ Current } & \multicolumn{1}{c|}{ Planned } \\
\hline 15 assembly line workers & 25 assembly line workers \\
\hline 25 sewing line workers & 30 sewing line workers \\
\hline 18 cutting line workers & 3 machines; 3 workers \\
\hline 5 quality checkers & 3 quality checkers \\
\hline Shoe TIS* $2 \frac{1}{2}$ hours & Shoe TIS* $1 \frac{1}{2}$ hours \\
\hline 225 pairs shoes per day & 492 pairs shoes per day \\
*TIS $=$ time in system
\end{tabular}

These results were summarized into direct answers of management's initial questions as follows:

Question: Should an investment be made in an automatic cutting machine?

Answer: Yes; this investment will permit the reduction of workers needed in the cutting line from 18 to 3 , as shown in Table 3 above.

Question: Does throughput increase?

Answer: Yes, even with fewer workers, it more than doubles, as shown in Table 3 above.

Question: Does the increased throughput from the cutting section affect other sections?

Answer: Yes, more workers will be needed in both the assembly and the sewing sections; these workers can be redeployed from the cutting line workers no longer needed.

Question: With fewer quality checkers, can quality be maintained? 
Answer: Yes, because the automated cutting machine will greatly reduce the error rate. However, it is acknowledged that the utilization of the three remaining quality checker will increase - expected to be a plus since inspection workers with too little to do may become bored and complacent - then miss defects (Ramzan, et al 2019)

Management, being highly pleased with and reassured by these results, is now beginning the implementation of machine investments and addition of labor, with conversion of manual transfer to conveyors to follow shortly and largely concurrently.

\section{CONCLUSIONS AND FUTURE WORK}

Given the promising results of this simulation analysis, ideas for follow-up work are already being considered. These ideas include refinement of the conveyor specifications (lengths, speeds, capacities, and nonaccumulating versus accumulating), expansion of the model to accommodate changes in demand mix (for example, one size may be more in demand than another), and adding analysis of different worker skill levels (hence payroll costs, pieces produced per hour, and reject rates).

\section{ACKNOWLEDGMENTS}

The authors gratefully acknowledge the cooperation and assistance of the manufacturing plant manager and workers in providing data, explaining details of the plant's processes and operation, and specifying the performance metrics of primary concern. Additionally, suggestions and criticisms from two anonymous referees have provided us significant help in improving this paper.

\section{REFERENCES}

Benneyan, C., 1998. Distribution fitting software makes simulation more attractive, viable in many applications. OR/MS Today (98:38,1-10)

Eryilmaz, M., Kuşakci, A., Gavranovic, H., and Findik, F., 2012. Southeast Europe Journal of Soft Computing (1:1, 120-127).

Mirzapourrezaei, S., Lalmazloumian, M., Dargi, A., and Wong, K., 2011. Simulation of a manufacturing assembly line based on WITNESS. Proceedings of the 2011 Third International Conference on Computational Intelligence, Communication Systems, and Networks, 132-137. July 26-28, Bali (Indonesia).

Mourtzis, D., Doukas, M., and Bernidaki, D., 2014. Simulation in manufacturing: Review and challenges. Proceedings of $8^{\text {th }}$ DET International Conference on Digital Enterprise Technology, 213229. March 25-28, Stuttgart (Germany).

Nakayama, M., 2003. Analysis of simulation output. Proceedings of the 2003 Winter Simulation Conference, Volume 1, 49-58. December 7-10, New Orleans, Louisana (United States of America).

Prochaska, K. and Thiesing, R., 2017. Introduction to Simio. Proceedings of the 2017 Winter Simulation
Conference, 4410-4419. December 3-6, Las Vegas, Nevada (United States of America).

Ramzan, M. B., Qureshi, S. M., Mari, S. I., Memon, M. S., Mittal, M., Imran, M., and Iqbal, M. W., 2019. Effect of time-varying factors on optimal combination of quality inspectors for offline inspection station. Mathematics (7:1, 51-68)

Sällström, J. and Schmidt, H., 1984. Cervicobrachial disorders in certain occupations, with special reference to compression in the thoracic outlet. American Journal of Industrial Medicine (6:1, 4552).

Smith, J., Sturrock, D., and Kelton, W., 2018. Simio and simulation: Modeling, analysis, applications. $5^{\text {th }}$ ed. Sewickley, Pennsylvania (United States of America): Simio LLC.

Solomon, H., Jilcha, K., and Berhan, E., 2014. Lead time prediction using simulation in leather shoe manufacturing. Advances in Intelligent Systems and Computing (334:283-292).

Sturrock, D. 2018., Avoid failures! Tested success tips for simulation product excellence. Proceedings of the 2018 Winter Simulation Conference, 252-260. December 2018, Göteborg (Sweden).

Weinswig, D., 2016. Sneaker culture fuels \$1 billion secondary market. 18 March, 22.

\section{AUTHOR BIOGRAPHIES}

Mohan Mano Hassan is a graduate student in Business Analytics enrolled with the college of Business at the University of Michigan-Dearborn. He completed a dual degree program at VIT University while earning his MBA. He has gained extensive experience in data analytics and data visualization while doing his Capstone Experience Project and Internship in Italy. $\mathrm{He}$ has undertaken projects studying the Flint (Michigan) water crisis, a market research project which enhanced his experience on various tools and technology. He has been awarded "Student of the Year" for his technical skills, leadership skills and helping the society. He helped small-scale and medium-scale companies reduce losses and increase profit with his Data Analysis and Management skills. He also earned a Merit award for his managerial skills while earning his MBA. His mail address is mohmano@umich.edu.

Sai Priyanka Kalamraju is a graduate student in Business Analytics enrolled with the College of Business at the University of Michigan-Dearborn. Prior to this, she studied Information Technology engineering as her first graduate degree program at Osmania University, India and pursued an MBA in Marketing from the Symbiosis Institute of Business Management, India. She has extensive experience in data analytics and data visualization with a keen eye for improving processes for companies large and small. She is a Research Assistant with the iLabs, a center for innovative research at the University of Michigan- Dearborn, where she uses data analysis to advise clients on real-time data. She has also 
had the opportunity of working with some of India's top companies, including Infosys and Kony Laboratories. At Kony Laboratories, she was instrumental in the automation process of weekly, monthly, quarterly, yearly reporting and helped in deriving metrics, KPIs regarding the profitability of the performance of the services. Due to her excellence in work, she also received the Spot award from the Executive Vice President and the Gem Team Award from Vice President in Kony Laboratories. She is a student member of the Indian Graduate Student Association (IGSA), Student Representative for the Winter Orientation program, and has worked with nonprofit organizations like Make a Difference, Shelter Homes, Children's Education, Plant 3 Protection Alliance on the plantation, environmental awareness, and Let's Vote on Voting awareness. She was also the recipient of the Non-Resident Graduate Tuition Differential Scholarship (NRTD) for Fall 2018. Her email address is kalamraj@umich.edu.

Venkata Sandeep Dangeti is a graduate student in Business Analytics enrolled with the college of Business at the University of Michigan-Dearborn. He came through a dual degree program; the first degree is MBA from VIT University in India and he is currently pursuing a second degree. Prior to that he founded a cashback website in India, where he got start-up exposure and practical experience, and where he diversified his entrepreneurial skills, managerial skills, technical skills, and leadership skills. He also worked in a multinational company (Tata Consultancy Services) where he learned how to work in a fast-paced environment. He worked with various tools and technology which made him learn quickly in any given situation. He consistently was in touch with stakeholders to improve and enhance software. He was awarded for a value-added service which saved the company considerable time and money. Apart from having professional experience in simulation, analytics and development, he also has Business Administration skills. He prepared many business plans for start-ups where he forecasted revenue, sales, demand and analyzed market trends. As a part of academic trips, he visited various industries in India and observed how small-scale, medium-scale and large-scale industries perform. He collaborated with many professionals and his friends in wide variety of projects. His email address is dvbittu@umich.edu.

Sirisha Pudipeddi is a graduate student pursuing her Master's in Science with a major in Business Analytics. With a computer science and engineering background, she has founded two start-ups in India. Her forte is in delivering business solutions with the right blend of information systems. She received the "Edex 40 under 40" award from The New Indian Express from the Information Technology Minister of the state of Telangana, India. She volunteers for a student organization named She's the First to help raise awareness and funds for educating girls in developing nations. Sirisha has an avid interest in the field of
Analytics and Project Management and is interning at the Global Product Development Center - Ford Motor Company, Dearborn. Her email address is sirishap@umich.edu.

Edward J. Williams holds bachelor's and master's degrees in mathematics (Michigan State University, 1967; University of Wisconsin, 1968). From 1969 to 1971, he did statistical programming and analysis of biomedical data at Walter Reed Army Hospital, Washington, D.C. He joined Ford Motor Company in 1972, where he worked until retirement in December 2001 as a computer software analyst supporting statistical and simulation software. After retirement from Ford, he joined PMC, Dearborn, Michigan, as a senior simulation analyst. Also, since 1980, he has taught classes at the University of Michigan, including both undergraduate and graduate simulation classes using GPSS $/ \mathrm{H}^{\mathrm{TM}}$, SLAM II ${ }^{\mathrm{TM}}$, SIMAN ${ }^{\mathrm{TM}}$, ProModel ${ }^{\circledR}$, SIMUL8 ${ }^{\circledR}$, or Arena ${ }^{\circledR}$. He is a member of the Institute of Industrial Engineers [IIE], the Society for Computer Simulation International [SCS], and the Michigan Simulation Users Group [MSUG]. He serves on the editorial board of the International Journal of Industrial Engineering - Applications and Practice. During the last several years, he has given invited plenary addresses on simulation and statistics at conferences in Monterrey, México; İstanbul, Turkey; Genova, Italy; Rīga, Latvia; and Jyväskylä, Finland. He served as a co-editor of Proceedings of the International Workshop on Harbour, Maritime and Multimodal Logistics Modelling \& Simulation 2003, a conference held in Rīga, Latvia. Likewise, he served the Summer Computer Simulation Conferences of 2004, 2005, and 2006 as Proceedings coeditor. He was the Simulation Applications track coordinator for the 2011 Winter Simulation Conference. A paper he co-authored with three of his simulation students won "best paper in track" award at the Fifth International Conference on Industrial Engineering and Operations Management, held in Dubai, United Arab Emirates, in March 2015. His email addresses are ewilliams@pmcorp.com and williame@umich.edu. 


\section{APPENDIX A}

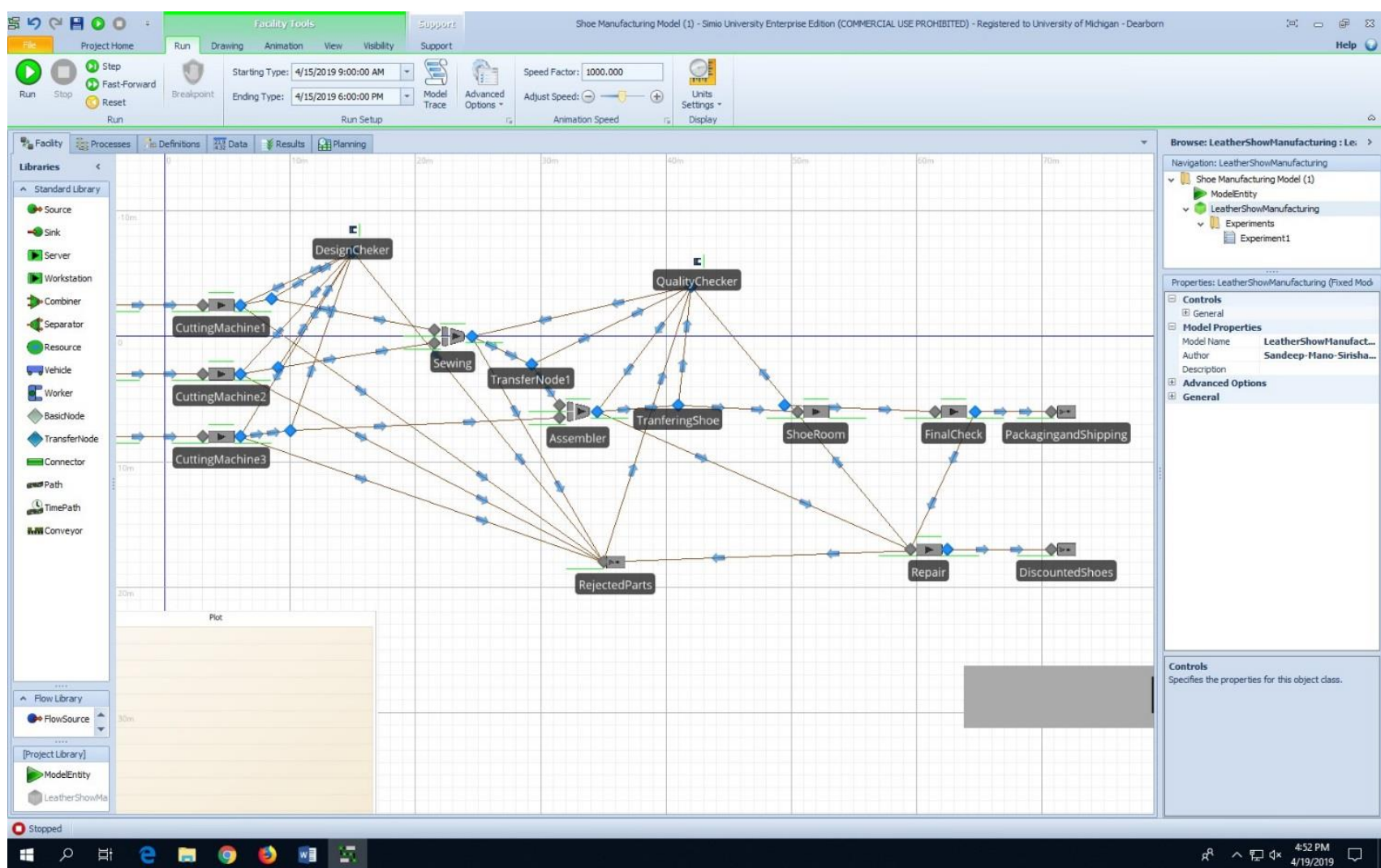

Figure 2: Screen of the Simio ${ }^{\circledR}$ Model Layout

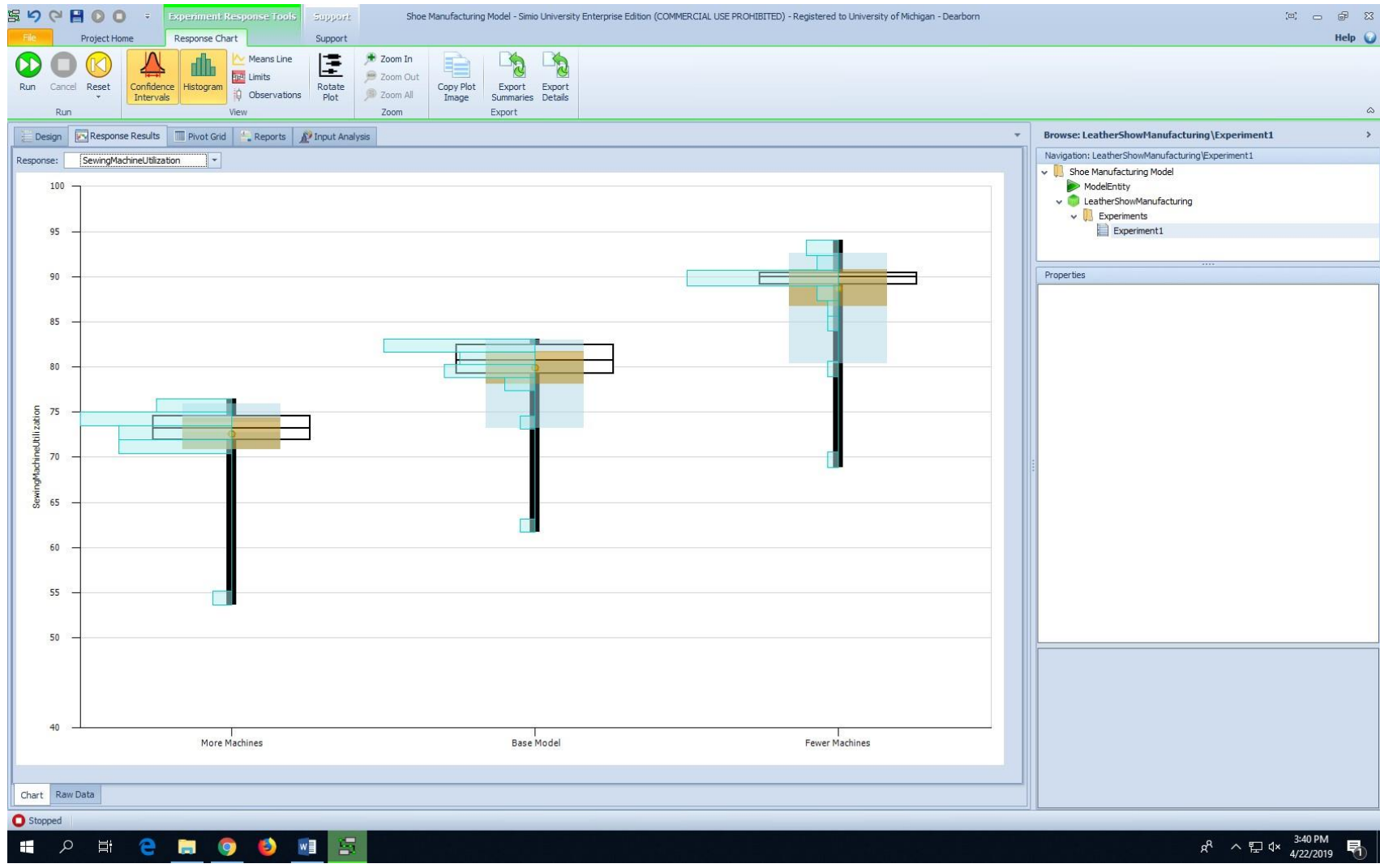

Figure 3: Example of Simio ${ }^{\circledR}$ Graphical Output Across Scenarios 